\title{
Supermembranes and Superstrings with Extrinsic Curvature
}

\author{
F. Aldabe* and A.L. Larsen"
}

October 8, 2018

Theoretical Physics Institute, Department of Physics, University of Alberta, Edmonton, Canada T6G 2J1

\begin{abstract}
In a recent paper Townsend suggested to associate to the $\mathrm{D}=11$ solitonic membrane of $\mathrm{N}=1$ supergravity a certain thickness, and then to identify this membrane with the fundamental supermembrane. By integrating out the 8 transverse dimensions of the "thick" solitonic membrane, we show that the resulting world-volume action indeed contains all the usual supermembrane terms, as well as background curvature terms and extrinsic curvature terms, which are believed to render the membrane spectrum discrete. We also outline the analog derivation for the "thick" $\mathrm{D}=10$ solitonic string solution of N=IIA supergravity. The resulting world-sheet action contains the usual type IIA superstring terms, as well as extra terms whose presence can be interpreted as a rescaling of the background metric, thus preserving kappa-symmetry and conformal invariance.
\end{abstract}

*Electronic address: faldabe@phys.ualberta.ca

"Electronic address: alarsen@phys.ualberta.ca 


\section{Introduction}

The supermembrane [1] seems to play a fundamental role in the unification of string theories, which are then interpreted as different perturbative expansions of the supermembrane. However, a major drawback of the supermembrane is that its spectrum is continuous [2], and therefore it cannot reproduce the particle spectrum we observe. The membrane has a continuous spectrum because no energy is required to create a spike of arbitrary height and of zero area [2]. Despite this, string-membrane duality in 10 dimensions between the type IIA superstring and the supermembrane requires that the type IIA fundamental string be identified with the solitonic string of $10 \mathrm{di}$ mensional $\mathrm{N}=\mathrm{IIA}$ supergravity, and that the supermembrane be identified with the solitonic membrane of 11 dimensional $\mathrm{N}=1$ supergravity [3].

As suggested in [3], this identification between fundamental and solitonic membrane can be used to yield a fundamental membrane which has a thickness. Spikes on this membrane will then necessarily have a non-zero area, and therefore energy will be required to create such spikes. Thus, a membrane with thickness is believed to have a discrete spectrum with a graviton multiplet as its ground state [四]. But we also must identify the fundamental and solitonic strings of the type IIA theory. Such an identification will lead to a fundamental string with a thickness. Alternatively, double dimensional reduction of the membrane [5] with a thickness, will also lead to a fundamental string with a thickness. It then seems that string-membrane duality along with discreteness of the supermembrane spectrum should lead to new fundamental string theories which contain thickness terms.

Here we derive to leading order the thickness terms of the supermembrane by integrating out the transverse dimensions of the solitonic membrane of 11 dimensional $\mathrm{N}=1$ supergravity. The thickness terms consist of background 
curvature terms as well as extrinsic curvature terms, which are believed to render the membrane spectrum discrete. We also derive to leading order the thickness terms for the type IIA superstring by integrating out the transverse dimensions of the solitonic string of 10 dimensional $\mathrm{N}=\mathrm{IIA}$ supergravity. We show that the fundamental string with thickness is equivalent to another string theory without thickness terms, but in a different vacuum where the background metric has acquired corrections of $\mathcal{O}\left(\alpha^{\prime}\right)$. In conclusion, we argue that the leading order thickness terms obtained by identifying the fundamental membrane (string) with the solitonic membrane (string) leads to a discrete spectrum for the membrane, but the identification does not lead to new string theories.

In Section 2 we review the solitonic membrane solution of 11 dimensional $\mathrm{N}=1$ supergravity. In Section 3 we derive the "thick" supermembrane from the solitonic membrane and explain why the thickness terms suppress the spikes. In Section 4 we derive the "thick" fundamental string from the solitonic string of the 10 dimensional N=IIA supergravity theory, and show how the thickness terms redefine the vacuum. Some concluding remarks are given in Section 5.

\section{The Solitonic Membrane of $\mathrm{D}=11$ Super- gravity}

The field equations in the bosonic sector of $\mathrm{N}=1$ supergravity in 11 dimensions, which can be derived from the action [6],

$$
\begin{aligned}
S_{11}=\frac{1}{2 \kappa^{2}} \int d^{11} Z[ & \sqrt{-g}\left(R-\frac{1}{48} F_{M N O P} F^{M N O P}\right) \\
& \left.+\frac{1}{12^{4}} \epsilon^{M N O P Q R S T U V W} F_{M N O P} F_{Q R S T} A_{U V W}\right],
\end{aligned}
$$


admit a "solitonic membrane" solution given by:

$$
\begin{gathered}
d s^{2}=\left(1+\frac{K}{r^{6}}\right)^{-2 / 3} \eta_{\mu \nu} d x^{\mu} d x^{\nu}+\left(1+\frac{K}{r^{6}}\right)^{1 / 3} \delta_{n m} d y^{n} d y^{m}, \\
A_{012}= \pm\left(1+\frac{K}{r^{6}}\right)^{-1},
\end{gathered}
$$

as was shown by Duff and Stelle [7]. Here $M, N=0,1, \ldots, 10 ; \mu, \nu=$ $0,1,2 ; m, n=3,4, \ldots 10$ and $r^{2} \equiv \delta_{n m} y^{n} y^{m}$. The membrane is parametrized by the constant $K$, which has dimension of $(\text { length })^{6}$, and which is taken to be positive.

Invariants constructed from $g_{M N}$ and $A_{M N P}$, such as $R, F^{2}, .$. are roughly stepfunctions. Indeed:

$$
R \sim F^{2} \sim \frac{K^{2}}{\left(K+r^{6}\right)^{7 / 3}}, \quad r>0 .
$$

Already at this stage, it is natural to associate to this membrane a certain "thickness", namely the thickness of the "tube" in which the invariants $R, F^{2}, .$. are significantly different from zero. In this sense, the thickness of the membrane (2.2)-(2.3), would be a number somewhat less than 1 in units of $K^{1 / 6}$. However, the solution (2.2)-(2.3) is only valid for $r>0$. To continue the solution to $r=0$, Duff and Stelle introduced a membrane-source in the supergravity field equations [7]. This leads to a consistent solution everywhere, but introduces (besides the source) a $\delta$-function singularity in the scalar curvature at $r=0$. However, as shown by Tseytlin [8, 9], one may expect that $\alpha^{\prime}$-corrections will smooth out the singularity and thus give rise to a finite thickness of the order of $\sqrt{\alpha^{\prime}}$. Duff, Gibbons and Townsend [10], on the other hand, suggested to avoid the source by analytical continuation of $r$ to imaginary values. They introduced a new radial coordinate $\hat{r} \equiv\left(r^{6}+K\right)^{1 / 6}$ and analytically continued it to $\hat{r}=0$. In that case, the $\delta$ function singularity at $r=0$ is exchanged by an ordinary Schwarzschild-like 
event horizon surrounding a physical singularity at $\hat{r}=0$ [10]. Now there is no problem of continuing the solution (2.2)-(2.3) through $r=0$; one merely has to express the solution in a coordinate system which is well-behaved at $r=0$. In particular, the expressions (2.4), which in fact are regular at $r=0$ anyway, can be continued through $r=0$ also.

In a recent paper [3], Townsend argued that one should associate to this analytically continued solitonic membrane a finite thickness of the order of the radius of the event horizon, i.e. again a thickness of the order $K^{1 / 6}$. He further suggested to identify this solitonic membrane with the fundamental supermembrane, which thereby acquires a finite thickness.

\section{Solitonic Membrane and Extrinsic Curva- ture}

The configuration (2.2)-(2.3) describes a flat static membrane, and it is the only explicitly known membrane solution of $\mathrm{N}=1$ supergravity in $\mathrm{D}=11$. In some sense it plays the same role as the flat static cosmic membrane, $\phi \sim$ $\tanh (z)$, in ordinary $\phi^{4}$-theory with Mexican hat potential, originally found by Zeldovich, Kobzarev and Okun [11. Inspired by this analogy and the work on generic curved dynamical cosmic membranes, see for instance [12], we will now be interested in the dynamics of a general curved solitonic membrane solution of the $\mathrm{N}=1$ supergravity field equations in $\mathrm{D}=11$.

The idea is to insert the solution for a generic curved solitonic membrane, which is of course not known explicitly in terms of $g_{M N}$ and $A_{M N P}$, into the action (2.1), and to integrate out the 8 transverse dimensions (in a first approximation, one may think of this procedure as considering small fluctuations around the flat static membrane). This will lead to an effective action determining the dynamics of the core of the solitonic membrane, and it is 
eventually to be identified with the world-volume action of the "thick" fundamental supermembrane. The procedure used to obtain this effective action is well-known from the theory of cosmic defects ( see for instance [12]), and it can be straightforwardly generalized to p-branes in supergravity. However, in previous discussions of p-branes in supergravity, the effective world-volume action has instead been postulated directly from its symmetries (see for instance 113, 14, 15, 16]), and therefore does not contain any knowledge about possible thickness terms. In the case of the solitonic membrane, where we do not want to erase completely the effects of the finite thickness, it is more appropriate to actually integrate out the transverse dimensions explicitly.

In order to perform the integration over the transverse dimensions, we will assume that the solitonic membrane under consideration is relatively smooth and slowly varying, in the sense that it locally looks like the flat static solution (2.2)-(2.3). It means that we will assume that all invariants constructed from $g_{M N}$ and $A_{M N P}$ are constant on the core of the membrane and that, close to the core, they only depend on the transverse coordinates. These assumptions are similar to the assumptions usually made for the Higgs field in cosmic membrane theory [12]; in our case, the role of the Higgs field is played by the background fields $g_{M N}$ and $A_{M N P}$ themselves.

It is convenient to define coordinates on the core of the membrane:

$$
Z^{M}\left(x^{\mu}, y^{m}\right)=X^{M}\left(x^{\mu}\right)+y^{m} N_{m}^{M}\left(x^{\mu}\right)
$$

and to introduce Riemann normal coordinates:

$$
g_{M N}=\eta_{M N}-\frac{1}{3} y^{n} y^{m} N_{n}^{P} N_{m}^{Q} R_{M P N Q}(y=0)+\mathcal{O}\left(y^{3}\right) .
$$

We use the same notation as in (2.2)-(2.3), i.e. $M, N=0,1, \ldots, 10 ; \mu, \nu=$ $0,1,2 ; \quad m, n=3,4, \ldots 10$. 
The normal vectors $N_{m}^{M}$ fulfill:

$$
\eta_{M N} X_{, \mu}^{M} N_{m}^{N}=0, \quad \eta_{M N} N_{n}^{N} N_{m}^{M}=\delta_{n m}
$$

as well as the completeness relation:

$$
\eta^{M N}=\gamma^{\mu \nu} X_{, \mu}^{M} X_{, \nu}^{N}+\delta^{n m} N_{m}^{M} N_{n}^{N}
$$

where $\gamma_{\mu \nu}$ is the induced metric on the core of the membrane:

$$
\gamma_{\mu \nu}=\eta_{M N} X_{, \mu}^{M} X_{, \nu}^{N}
$$

From (3.3)-(3.4) it can be shown that the normal vectors $N_{m}^{M}$ are functions of $X^{M}$ but not of $X_{, \mu}^{M}$, in the sense that the transformation laws under worldvolume reparametrizations are given by:

$$
\delta X^{M}=\xi^{\mu} X_{, \mu}^{M}, \quad \delta N_{m}^{M}=\xi^{\mu} N_{m, \mu}^{M} .
$$

We also define the extrinsic curvature $K_{\mu \nu n}$ and torsion $\omega_{m n \nu}$ :

$$
K_{\mu \nu n}=\eta_{M N} X_{, \mu}^{M} N_{n, \nu}^{N}, \quad \omega_{m n \nu}=\eta_{M N} N_{m}^{M} N_{n, \nu}^{N} .
$$

The idea is now to integrate out the 8 transverse $y$-coordinates in the action (2.1). The simplest way to do this, is to Taylor-expand the integrand in powers of $y$, and to perform the integration only for "small" $y(\sim$ one unit in the thickness of the membrane). This is a reasonable approximation of the actual integral, provided the integrand resembles a "narrow" stepfunction. This is actually the case for the flat static membrane (2.2)-(2.3), and we can expect this to hold also for a more general smoothly curved and slowly varying membrane.

Let us first consider the volume element in (2.1). It is a standard exercise to compute the metric $g_{M N}$ in the $(x, y)$-coordinates:

$$
\begin{aligned}
g_{\mu \nu}=\gamma_{\mu \nu} & +2 y^{m} K_{\mu \nu m}+y^{n} y^{m}\left[K_{\mu \rho m} K_{\nu}{ }^{\rho}{ }_{n}+\omega_{p m \mu} \omega^{p}{ }_{n \nu}\right. \\
& \left.-\frac{1}{3} N_{m}^{P} N_{n}^{Q} X_{, \mu}^{M} X_{, \nu}^{N} R_{M P N Q}(y=0)\right]+\mathcal{O}\left(y^{3}\right),
\end{aligned}
$$




$$
\begin{gathered}
g_{\mu m}=y^{n} \omega_{m n \mu}-\frac{1}{3} y^{n} y^{p} N_{n}^{P} N_{p}^{Q} N_{m}^{N} X_{, \mu}^{M} R_{M P N Q}(y=0)+\mathcal{O}\left(y^{3}\right), \\
g_{m n}=\delta_{m n}-\frac{1}{3} y^{p} y^{q} N_{p}^{P} N_{q}^{Q} N_{m}^{M} N_{n}^{N} R_{M P N Q}(y=0)+\mathcal{O}\left(y^{3}\right) .
\end{gathered}
$$

It follows that:

$$
\begin{aligned}
\sqrt{-g}=\sqrt{-\gamma}\left[1+y^{n} K_{\mu n}^{\mu}\right. & +\frac{1}{2} y^{n} y^{m}\left(K_{\mu n}^{\mu} K_{\nu m}^{\nu}-K^{\mu \nu}{ }_{n} K_{\mu \nu m}\right. \\
& \left.\left.-\frac{1}{3} N_{m}^{M} N_{n}^{N} R_{M N}\right)+\mathcal{O}\left(y^{3}\right)\right] .
\end{aligned}
$$

Next we consider the Lagrangian. As already explained, we assume that all invariants $\left(R, F^{2}, \ldots\right)$ constructed from $\left(g_{M N}, A_{M N P}\right)$ are constant on the core of the membrane, and close to the core, they only depend on the $y$-coordinates. It should be stressed that these assumptions are not made to simplify the mathematics; they are merely conditions for the actual existence of membrane-shaped solutions of the $D=11$ supergravity field equations. Thus, considering a membrane which locally resembles the flat static membrane (2.2)-(2.3), we can assume:

$$
\begin{gathered}
R=R(y=0)+\mathcal{O}\left(y^{2}\right), \\
F^{2}=F^{2}(y=0)+\mathcal{O}\left(y^{2}\right) .
\end{gathered}
$$

These expressions will be valid for small $y$, that is, inside the range of the $y$-integration, c.f. the comments after equation (3.7). Notice also that the assumptions (3.12)-(3.13) are relatively "modest" for a smooth and slowly varying membrane. For the flat static membrane (2.2)-(2.3), the first corrections to the constant values at $y=0$ are actually of $\mathcal{O}\left(y^{6}\right)$.

Generally there is no reason for $g_{M N}$ and $A_{M N P}$ to be functions of the $y$-coordinates only (although this is the case for the flat static membrane (2.2)-(2.3)). Contrary to $\left(R, F^{2}, \ldots\right)$, they are not invariants and therefore will depend on the specifically chosen coordinates and three-form gauge. 
However, to fulfill equation (3.13), $A_{M N P}$ must have the form:

$$
A_{M N P}:\left\{\begin{array}{l}
A_{\mu \nu \rho}=A_{\mu \nu \rho}(X(x))+\mathcal{O}\left(y^{3}\right) \\
A_{\mu \nu m}=A_{\mu m n}=\mathcal{O}\left(y^{3}\right) \\
A_{m n p}=y^{q} C_{m n p q}+\mathcal{O}\left(y^{3}\right)
\end{array}\right.
$$

where $C_{m n p q}$ are constants, while $A_{\mu \nu \rho}$ are arbitrary functions of $X$. It follows that:

$\epsilon^{M N O P Q R S T U V W} F_{M N P Q} F_{Q R S T} A_{U V W} \sim \epsilon^{\mu \nu \rho} X_{, \mu}^{M} X_{, \nu}^{N} X_{, \rho}^{P} A_{M N P}(y=0)+\mathcal{O}\left(y^{2}\right)$.

Thus, the topological term of 11 dimensional $\mathrm{N}=1$ supergravity is responsible for the Wess-Zumino term of the supermembrane action.

Using also the following integrals:

$$
\int^{\prime} d^{8} y \sim K^{4 / 3}, \quad \int^{\prime} y^{n} d^{8} y=0, \quad \int^{\prime} y^{n} y^{m} d^{8} y \sim K^{5 / 3} \delta^{n m},
$$

where the prime denotes integration only over the "thickness" ( one unit in $K^{1 / 6}$ ), we get the following result from (2.1) to zeroth order in $y$ (after a constant redefinition of $A_{M N P}$ and $\left.g_{M N}\right)$ :

$$
S_{M}^{(0)} \sim \frac{K}{\kappa^{2}} \int d^{3} x\left[\sqrt{-\gamma}+\epsilon^{\mu \nu \rho} X_{, \mu}^{M} X_{, \nu}^{N} X_{, \rho}^{P} A_{M N P}\right]
$$

that is, the usual bosonic sector of the supermembrane action [1]. In cosmic membrane theory, this would describe the zero-thickness limit [12]. However, for the solitonic membrane it has no physical meaning to take this limit because of the singularity in the center of the core. In other words, we must consider a membrane of finite thickness or equivalently of finite $K$. This corresponds to keeping terms of higher order in $y$ in the action (2.1). To second order in $y$, we get from (2.1):

$$
S_{M}^{(2)} \sim \frac{K^{4 / 3}}{\kappa^{2}} \int d^{3} x \sqrt{-\gamma}\left[K_{\mu n}^{\mu} K_{\nu}{ }^{\nu n}-K^{\mu \nu}{ }_{n} K_{\mu \nu}{ }^{n}-\frac{\delta^{n m}}{3} R_{M N} N_{m}^{M} N_{n}^{N}\right]
$$


that is, the extrinsic curvature terms as well as a term due to the curvature of the $\mathrm{D}=11$ background. Notice that to this order in $y$, the corrections (3.18) come entirely from the Jacobian of the coordinate transformation (3.1), that is to say, from equation (3.11). To higher orders there will be corrections also from the Lagrangian, and in particular, there will be corrections to the Wess-Zumino term.

It must be stressed that the action (3.17)-(3.18) has been derived only for very special $\mathrm{D}=11$ backgrounds, namely backgrounds that admit membraneshaped solutions to the $\mathrm{D}=11$ supergravity field equations. However, to identify (3.17)-(3.18) with the action of a new "thick" fundamental supermembrane, we extrapolate the result to arbitrary $\left(g_{M N}, A_{M N P}\right)$. It is important that the extrinsic curvature terms in (3.18) survive, even in the background of Minkowski space. Extrinsic curvature terms have been discussed in other areas of physics, and are generally known to suppress "spikes", see for instance [17], thus it is reasonable to believe that the spectrum, as obtained (non-perturbatively) from (3.17)-(3.18), will be discrete. Notice also that in the background of Minkowski space, by using the Gauss-Codazzi equation [18], the action (3.18) reduces to:

$$
S_{M}^{(2)} \sim \frac{K^{4 / 3}}{\kappa^{2}} \int d^{3} x \sqrt{-\gamma}^{(3)} R(\gamma)
$$

where ${ }^{(3)} R(\gamma)$ is the scalar curvature of the membrane. This means that even in flat Minkowski space a thickness term is present to insure that the spectrum of the supermembrane is discrete.

A question that must be addressed is the preservation of supersymmetry and kappa-symmetry after including the leading order thickness terms [19, 20]. For simplicity we treat only the case in which the target space is flat. Although supersymmetry is not apparent in the action obtained from the solitonic membrane, it is a standard procedure to construct the 
supersymmetric version, see for instance [1]. The expression (3.19) will then be invariant under the supersymmetry transformations of [4], which leave the leading order supermembrane action (3.17) invariant. As far as kappasymmetry is concerned, to leading order the action is kappa-invariant [ [ 1 ]. This means that to leading order the spectrum is supersymmetric. However, the presence of (3.19) may lead to kappa-symmetry breaking (unless some compensating terms can be added) and therefore to a supersymmetry breaking of the particle spectrum. We hope to address this issue somewhere else; see also the comments in the Conclusion.

\section{Solitonic String and the Rescaled Metric}

The solitonic string solution in 10 dimensions [14 was originally constructed from the $\mathrm{N}=1$ supergravity field equations. For our purposes, it is however convenient to consider the full $\mathrm{N}=\mathrm{IIA}$ supergravity action in $\mathrm{D}=10$ :

$$
\begin{gathered}
S_{10}=\frac{1}{2 \kappa^{2}} \int d^{10} Z\left[\sqrt{-g}\left\{e^{-2 \Phi}\left(R+4(\nabla \Phi)^{2}-\frac{1}{3} H^{2}\right)-G^{2}-\frac{1}{12} \tilde{F}^{2}\right\}\right. \\
\left.-\frac{1}{288} \epsilon^{M N O P Q R S T U V} F_{M N O P} F_{Q R S T} B_{U V}\right]
\end{gathered}
$$

from which the $\mathrm{N}=1$ supergravity action is obtained by truncation, and we consider only the bosonic part. Here $\Phi$ is the dilaton, $G=d A, H=d B, F=$ $d C$ and $\tilde{F}=d C+2 A \wedge H$ ( $A$ is the one-form, $B$ the two-form and $C$ the three-form). The solitonic string solution [14] is then given by:

$$
\begin{gathered}
A=0, \quad C=0 \\
d s^{2}=\left(1+\frac{K}{r^{6}}\right)^{-3 / 4} \eta_{\mu \nu} d x^{\mu} d x^{\nu}+\left(1+\frac{K}{r^{6}}\right)^{1 / 4} \delta_{n m} d y^{n} d y^{m} \\
B_{01}= \pm\left(1+\frac{K}{r^{6}}\right)^{-1}, \quad e^{\Phi}=\left(1+\frac{K}{r^{6}}\right)^{-1 / 2}
\end{gathered}
$$


and we use the similar notation as in (2.1)-(2.3): $M, N=0,1, \ldots, 9 ; \mu, \nu=$ 0,$1 ; m, n=2,3, \ldots 9$. Notice also that the line element (4.3) is expressed in Einstein frame:

$$
g_{M N}^{\text {String }}=e^{\Phi / 2} g_{M N}^{\text {Ein }}
$$

As for the solitonic membrane, there is a $\delta$-function singularity at $r=0$, corresponding to the string-source. However, by a construction following the steps of the membrane-case, but involving also a rescaling of the metric and a reinterpretation of the dilaton [10], the source can be removed by analytical continuation beyond $r=0$. Again it is found that $r=0$ becomes a mere coordinate singularity (a horizon) surrounding a true curvature singularity in the analytically continued metric. Thus the situation is very similar to the case of the membrane. The solitonic string (4.2)-(4.4) is the straight static string-shaped solution to the field equations obtained from the action (4.1). Following the derivation of Section 3, it is then straightforward to obtain the effective world-sheet action describing the core of a more general curved non-static string solution, so we just present the results here without going into the computational details.

The analog of (3.17) becomes:

$$
S_{S}^{(0)} \sim \frac{K}{\kappa^{2}} \int d^{2} x\left[\sqrt{-\gamma}+\epsilon^{\mu \nu} X_{, \mu}^{M} X_{, \nu}^{N} B_{M N}\right],
$$

that is, the usual bosonic sector of the superstring action. Notice that the WZ-term was obtained from the $F \wedge F \wedge B$-term in (4.1), by a decomposition of $B$ similar to (3.14). To second order in $y$ we get the terms:

$$
S_{S}^{(2)} \sim \frac{K^{4 / 3}}{\kappa^{2}} \int d^{2} x \sqrt{-\gamma}\left[W(X)+{ }^{(2)} R(\gamma)\right]
$$

where:

$$
W(X)=\frac{5}{3} \delta^{n m} R_{M N} N_{m}^{M} N_{n}^{N}-\delta^{m p} \delta^{n q} R_{M N P Q} N_{m}^{M} N_{n}^{N} N_{p}^{P} N_{q}^{Q},
$$


and we have used the Gauss-Codazzi equation [18].

We should stress, that as in the case of the membrane, it makes no sense to take the zero thickness limit (and thus get rid of (4.7)) because of the singularity in the center of the string core. As in the membrane-case, we now take the action (4.6)-(4.7) and extrapolate it to arbitrary $\left(g_{M N}, B_{M N}\right)$. In Minkowski space we just get the usual type IIA superstring action (bosonic sector), while the $W(X)$-term in (4.7) must be taken into account in a general curved background.

Let us consider (4.6)-(4.7) in a little more detail. The second term in (4.7) is topological and therefore can be skipped, because the action is twodimensional. Furthermore, introduce the string tension:

$$
\frac{K}{\kappa^{2}} \sim \frac{1}{\alpha^{\prime}}, \quad \kappa^{2} \sim\left(\alpha^{\prime}\right)^{4}
$$

and write the action (4.6)-(4.7) in "Polyakov" form:

$$
\begin{aligned}
S_{S} & \sim S_{0}+\alpha^{\prime} S_{1} \\
S_{0} & =\frac{1}{\alpha^{\prime}} \int d^{2} x\left[\sqrt{-h} h^{\mu \nu} \eta_{M N} X_{, \mu}^{M} X_{, \nu}^{N}+\epsilon^{\mu \nu} X_{, \mu}^{M} X_{, \nu}^{N} B_{M N}\right] \\
S_{1} & =\frac{1}{\alpha^{\prime}} \int d^{2} x \sqrt{-h} h^{\mu \nu} \eta_{M N} W(X) X_{, \mu}^{M} X_{, \nu}^{N}
\end{aligned}
$$

where $h_{\mu \nu}$ is the Lagrange multiplier field.

The term $S_{1}$ simply rescales the background metric by terms of $\mathcal{O}\left(\alpha^{\prime}\right)$. A term which seems to be missing is the dilaton term, ${ }^{(2)} R(\gamma) \Phi$. A similar situation was encountered in [21], where the world-sheet action without thickness terms for the heterotic string was derived. At first one would expect the conformal invariance to be broken. However, the dilaton term appears by considering an effective world-sheet action which preserves conformal invariance. This is equivalent to adding the dilaton term in order to insure that the $\beta$-functions vanish, thus restoring conformal invariance. Since the 
term $S_{1}$ only rescales the metric, it is always possible to find a background dilaton which will insure that the $\beta$-functions vanish. In terms of the background dilaton, $\Phi_{s}$, which defines the solitonic string, we may define the new background dilaton which restores conformal invariance

$$
e^{2 \Phi_{n e w}}=e^{2 \Phi_{s}}\left[1+\alpha^{\prime} W(X)\right]
$$

The constraints imposed on the background antisymmetric tensor to achieve kappa-symmetry are thus compatible with the vanishing of the $\beta$-functions [22].

\section{Concluding Remarks}

The main obstacle to accept the supermembrane to describe the observed particle spectrum is that its spectrum is continuous. We have shown, as suggested by Townsend and needed by string-membrane duality, that the identification between the solitonic and fundamental membrane yields a supermembrane action with thickness terms, which are believed to suppress the creation of spikes of zero area, and therefore render the spectrum discrete. In addition, as required by the $E_{7}(Z)$ invariance of the spectrum [23] of the type IIA superstring and by string-membrane duality, we must also identify the solitonic string with the fundamental string. We have shown that this identification does not yield a new string theory, but rather that it leads to a redefinition of the vacuum, at least when including only the lowest order thickness terms.

An open problem on which we hope to report elsewhere, is the possible breaking of kappa-invariance and thus of the supersymmetry of the particle spectrum of the membrane, due to thickness terms. If kappa-invariance is broken, it is only softly broken in the sense that the kappa-symmetry breaking 
terms will be of $\mathcal{O}\left(\alpha^{\prime}\right)$. This would mean that the requirement of stringmembrane duality not only yields a supermembrane with a discrete spectrum but also a spectrum which has a softly broken supersymmetry. Thus, this might be the much sought soft supersymmetry-breaking mechanism in stringmembrane theory.

\section{Acknowledgements}

The work of A.L. Larsen was supported by NSERC. F. Aldabe would like to thank B. Campbell for discussion and W. Israel for ecouragement and support. We also thank A. Tseytlin for drawing our attention to Refs. [8, 9].

\section{References}

[1] E. Bergshoeff, E. Sezgin, and P.K. Townsend, Phys. Lett. B198 (1987) 75.

[2] B. De Wit, M. Luscher and H. Nicolai, Nucl.Phys. B320 (1989) 135.

[3] P.K. Townsend, Phys. Lett. B350 (1995) 184.

[4] E. Bergshoeff, E. Sezgin and P.K. Townsend, Ann. Phys. 185 (1988) 330.

[5] M.J. Duff, P.S. Howe, T. Inami and K.S. Stelle, Phys. Lett. B191 (1987) 70.

[6] W. Nahm, Nucl.Phys. B135 (1978) 149; E. Cremmer, B. Julia, J. Scherk, S. Ferrara, L. Girardello and P. Van Nieuwenhuizen, Nucl.Phys. B147 (1979) 105. 
[7] M.J. Duff and K.S. Stelle, Phys. Lett. B253 (1991) 113.

[8] A. Tseytlin, Phys. Lett. B363 (1995) 223.

[9] A. Tseytlin, "Selfduality of Born-Infeld Action and Dirichlet 3-Brane of the Type IIB Superstring Theory", Imperial-TP-95-96-26, hepth/9602064.

[10] M.J. Duff, G.W. Gibbons and P.K. Townsend, Phys.Lett. B332 (1994) 321.

[11] Y.B. Zeldovich, I.Y. Kobzarev and L.B. Okun, Sov. Phys. JETP 40 (1975) 1.

[12] D. Garfinkle and R. Gregory, Phys. Rev. D41 (1990) 1889; R. Gregory, D. Haws and D. Garfinkle, Phys. Rev. D42 (1990) 343; R. Gregory, Phys. Rev. D43 (1991) 520.

[13] J. Hughes, J. Liu and J. Polchinski, Phys. Lett. B180 (1986) 370.

[14] A. Dabholkar, G.W. Gibbons, J.A. Harvey and F. Ruiz-Ruiz, Nucl. Phys. B340 (1990) 33.

[15] C.G. Callan, J.A. Harvey and A. Strominger, Nucl. Phys. B367 (1991) 60 .

[16] M.J. Duff and J.X. Lu, Phys. Rev. Lett. 66 (1991) 1402.

[17] H. Kleinert, Phys. Lett. B174 (1986) 335; A.M. Polyakov, Nucl. Phys. B268 (1986) 406; H. Koibuchi, Phys. Lett. B242 (1990) 371.

[18] L.P. Eisenhart, Riemannian Geometry (Princeton University Press, fifth printing, 1964). 
[19] T. Curtright and P. van Nieuwenhuizen, Nucl Phys. B294 (1987) 125.

[20] J.P. Gauntlett, K. Itoh and P.K. Townsend, Phys. Lett. B238 (1990) 65.

[21] A. Dabholkar, Phys. Lett. B357 (1995) 307.

[22] M.T. Grisaru, P. Howe, L. Mezincescu, B.E.W. Nilsson and P.K. Townsend, Phys. Lett. B162 (1985) 116.

[23] C.M. Hull and P.K.Townsend, Nucl. Phys. B438 (1995) 109. 